\title{
The impact of biologic therapy in chronic plaque psoriasis from a societal perspective: an analysis based on Italian actual clinical practice
}

\author{
B. Polistena, ${ }^{1, \star}$ P. Calzavara-Pinton, ${ }^{2}$ G. Altomare,${ }^{3}$ E. Berardesca, ${ }^{4}$ G. Girolomoni, ${ }^{5}$ P. Martini, ${ }^{6}$ \\ A. Peserico, ${ }^{7}$ A. Puglisi Guerra, ${ }^{8}$ F. Spandonaro, ${ }^{1}$ A. Vena Gino, ${ }^{9}$ S. Chimenti, ${ }^{10}$ F. Ayala ${ }^{11}$ \\ ${ }^{1}$ Tor Vergata University of Rome, Rome, Italy \\ ${ }^{2}$ Department of Dermatology, Spedali Civili, Brescia, Italy \\ ${ }^{3}$ Department of Dermatology, University of Milan, Milan, Italy \\ ${ }^{4}$ San Gallicano Dermatological Institute, Rome, Italy \\ ${ }^{5}$ Department of Medicine, Dermatology and Venereology Sector, University of Verona, Verona, Italy \\ EUnit of Dermatology, Lucca Hospital, Lucca, Italy \\ ${ }^{7}$ Unit of Dermatology, Department of Medicine, University of Padua, Padua, Italy \\ ${ }^{8}$ Department of Dermatology, Messina Hospital, Messina, Italy \\ ${ }^{9}$ Unit of Dermatology and Venereology, University of Bari, Bari, Italy \\ ${ }^{10}$ Department of Dermatology, Tor Vergata University of Rome, Rome, Italy \\ ${ }^{11}$ Department of Dermatology, Federico II University of Naples, Naples, Italy \\ *Correspondence: B. Polistena. E-mail: barbara.polistena@uniroma2.it
}

\begin{abstract}
Objective Psoriasis is one of the most common forms of chronic dermatitis, affecting 2-3\% of the worldwide population. It has a serious effect on the way patients perceive themselves and others, thereby prejudicing their quality of life and giving rise to a significant deterioration in their psycho-physical well-being; it also poses greater difficulties for them in leading a normal social life, including their ability to conduct a normal working life. All the above-mentioned issues imply a cost for the society. This study proposes to evaluate the impact on societal costs for the treatment of chronic plaque psoriasis with biologics (etanercept, infliximab and adalimumab) in the Italian clinical practice.

Method A prospective observational study has been conducted in 12 specialized centres of the Psocare network, located throughout Italy. Direct and indirect costs (as well as the health-related quality of life of patients with plaque psoriasis undergoing biologic treatments) have been estimated, while the societal impact has been determined using a cost-utility approach. Results Non-medical and indirect costs account for as much as $44.97 \%$ of the total cost prior to treatment and to $6.59 \%$ after treatment, with an overall $71.38 \%$ decrease. Adopting a societal perspective in the actual clinical practice of the Italian participating centres, the ICER of biologic therapies for treating plaque psoriasis amounted to $€ 18634.40$ per QALY gained - a value far from the $€ 28656$.30 obtained by adopting a third-party payer perspective.

Conclusion Our study confirms that chronic psoriasis subjects patients to a considerable burden, together with their families and caregivers, stressing how important it is to take the societal perspective into consideration during the appraisal process. Besides, using data derived from Italian actual practice, treatment with biologics shows a noteworthy benefit in social terms.

Received: 25 February 2015; Accepted: 18 May 2015

\section{Conflicts of interest}

GG has received lecture and/or consultation fees from Pfizer, Actelion, Novartis, Abbvie, MSD, Almirall and Celgene. AP has received fees as speaker or consultant or has received research or educational support for the Dermatology Unit of the Department of Medicine of the University of Padua from Abbott, Almirall, MSD, JanssenCilag, Pfizer, GSK and Leo Pharma. FS has received research and educational grants from Abbott, Bayer, Biogen Idec, Biomarine, BMS, and Boehringer-Ingelheim, Celgene, Daiichy Sankyo, Eli Lilly, Genzyme, GlaxoSmithKline, Janssen Cilag, Johnson \& Johnson, Medtronic, MSD Italia, Novartis, Novo Nordisk, Obi, Pfizer, Roche, Sanofi Pasteur MSD, Servier, Sigma Tau, Stroder and Teva. GAV has been a speaker, a consultant and/or a member of the Advisory Board for Abbvie, MSD, Novartis, Pfizer, Janssen-Cilag and Leo Pharma. SC has been a consultant and/or a speaker for Merck, Pfizer, Abbvie, Novartis, Janssen-Cilag and Leo Pharma.
\end{abstract}




\section{Introduction}

Psoriasis is a common inflammatory skin disease, with a chronic relapsing-remitting course, characterized by scaly, red patches most commonly on the elbows, knees, hands, feet and scalp. Several forms of psoriasis have been classified, but the most common form is plaque psoriasis, affecting about $80 \%$ of psoriatic patients. ${ }^{1}$ Given its symptoms, psoriasis may have significant psychological effects on a patient's self-esteem, body perception and sense of well-being that reach far beyond the physical aspects. $^{2-4}$ In fact, psoriasis has a substantial negative impact on all aspects entailing the quality of life, including physical and psychological factors, social relationships, as well as employment - resulting in a significant economic burden on patients, healthcare systems and society. ${ }^{15}$

Economic impact in terms of direct medical costs (i.e. adopting the third-party payer perspective) has been largely investigated by resorting to economic modelling ${ }^{2-6}$ and, recently, even by analysing cost-effectiveness in the actual practice. ${ }^{16}$ Adopting a societal perspective, costs associated with work-related absent or impaired productivity for patients and their family members, travel costs incurred when going to medical appointments, costs associated with domestic support, and other non-medical costs might also represent a significant share of the total cost associated with psoriasis.

Many studies present strong evidence for substantial indirect costs imposed by psoriasis not only on patient affected by the disease, but also on their partners, family members and/or caregivers. $^{2,5,7-9}$

However, most analyses do not consider the impact of biologic agents since these studies were conducted before biologics became available for the treatment of psoriasis.

The aim of this study is to quantify (through a representative caseload of patients, seen in a clinical practice setting of Italian specialist centres) the impact of biologic agents on direct and indirect non-medical cost, also evaluating cost-effectiveness of biologic therapy for the treatment of chronic plaque psoriasis from a societal perspective. The study provides new insight into the economic evaluation of societal costs of chronic plaque psoriasis, employing evidence from the utilization of biologic agents in an actual clinical practice.

\section{Methods}

\section{Study design}

A prospective observational study was conducted in a 'realworld' clinical practice setting in Italy, with a view towards determining medical and non-medical direct costs, as well as indirect costs and the health-related quality of life (HQoL) of patients suffering from chronic plaque psoriasis. The study was conducted in 12 specialized centres belonging to the Psocare network throughout Italy. During the period from 11 May 2009 until 31 December 2009, all the adult psoriatic patients switching to biologic therapy, or those who reverted to biologic treatment after a suspension of at least 1 year, were enrolled in the study (more details in Ref. 16). The only biologic agents approved in Italy for the treatment of psoriasis at the time of the study were etanercept, infliximab and adalimumab.

The Psocare project, designed to evaluate the long-term effectiveness and safety of available psoriasis treatments, is an initiative promoted by the Italian Medicines Agency (AIFA) in conjunction with dermatology societies and patient associations, under the technical coordination of the GISED (Italian Group for Epidemiologic Research in Dermatology) research centre. Psocare conducts its work based on the philosophy that psoriasis treatment strategies devised to date have led to the consolidation of habits or behaviours amongst doctors, instead of striving for clear outcomes in terms of effectiveness. Therefore, comparisons between different care strategies have been made with a view towards obtaining a realistic estimate of benefits and risks, with the intention of providing important data for the evaluation of the outcome of the treatments provided to psoriatic patients.

In compliance with the Declaration of Helsinki protocols, appropriate consent was obtained from all the participants. As the study was a non-interventional observation study and treatment, diagnostic procedures and follow-up were representative of current actual clinical practice, there was no need for formal ethical approval from individual Ethics Committees.

\section{Cost, quality of life and clinical assessments}

Upon enrolment in the study, the costs incurred prior to switching to biologic therapy were calculated in retrospect; besides patient HQoLs and objective/subjective clinical conditions were evaluated at baseline using the validated scales devised specifically for psoriasis (the Psoriasis Area Severity Index [PASI] and pain and itching Visual Analogue Scales [VAS]). The questionnaires and data collection procedures were repeated at the end of a 6-month follow-up period.

Quality of life was measured using the five-dimension European Quality of Life Questionnaire (EuroQoL-5D or EQ-5D); objective severity of the condition was measured using PASI scores; while pain and itching VAS scores were used to get to a subjective evaluation of the clinical conditions of patients. The direct medical costs included all costs incurred in connection with psoriasis: hospitalization, day hospital and/or outpatient services, specialist appointments, laboratory tests, diagnostic procedures, phototherapy and medication (more details in Ref. 16).

The direct non-medical costs taken into consideration included the time spent for visits and the need for help in performing household chores. The latter was calculated using the effective minimum hourly wage of $€ 7.22$, according to Italian Institute of Social Security criteria. Italian Automobile Club vehicle running 
cost data for a medium-sized hatchback were used when calculating travel costs, assuming a unit cost per $\mathrm{km}$ of $€ 0.30$.

The number of days away from work was also recorded to calculate productivity loss, in keeping with the human capital approach. The average number of days with reduced working capacity was also recorded. A record was also made of the number of days patients were unable to make full use of their leisure time and those in which they required help from their family members.

Questionnaires were administered using the CAPI (Computer-Assisted Personal Interviewing) method. Those completed by patients included general details and socioeconomic data, the EuroQoL-5D questionnaire, in addition to retrospective information on the healthcare resources used in connection with psoriasis (medications, visits, diagnostics, day hospital and/or outpatient services, information needed to estimate indirect and direct non-medical costs) before enrolment. Those completed by doctors involved the main clinical evaluation elements (PASI, pain VAS and itching VAS) and medication-related information.

\section{Cost-utility analysis}

A cost-utility approach was adopted, calculating the incremental cost-effectiveness ratio (ICER), comparing the QALY gained between baseline and follow-up with incremental direct/indirect costs.

\section{Results}

The analysis was conducted on 178 patients with a mean age of 47.7 years. The baseline characteristics of the patient caseload are shown in Table 1. All patients completed the follow-up period and were available for analysis. Although literature suggests that prevalence is similar in both genders, ${ }^{13}$ in this study males were prevalent, accounting for $64.6 \%$ of the total caseload. Altogether, $56.7 \%$ of patients enrolled were eligible for Italian National Health System (NHS) co-payment exemptions on account of their illness.

At the beginning of the study, $59.6 \%$ of patients were prescribed etanercept, $32.0 \%$ adalimumab and $8.4 \%$ infliximab.

Table 2 illustrates the clinical benefits and HQoL data recorded during the observation period. A general reduction in disease severity was assessed by the PASI score throughout the 6-month observation period; this was accompanied by improvement in pain parameters, itching parameters and improvement in HQoL.

In short, prior to enrolment, mean direct medical costs borne by the NHS were $€ 2166.20$ per patient on a yearly basis. With the switch to biologic therapy, these costs rose to $€ 15073.70$ per annum, with a 596.6\% increase. A more detailed analysis of direct cost data has been reported in Ref. 16.

Prior to enrolment, patients personally spent an additional $€ 928.20$ a year for appointments, diagnostic procedures and laboratory tests. Following the switch to biologic therapy, this figure
Table 1 Caseload characteristics at enrolment $(N=178)$

\begin{tabular}{|c|c|}
\hline Variable & Value \\
\hline Mean age, years (range) & $47.7(18-79)$ \\
\hline Mean age at diagnosis, years & 30.6 \\
\hline Males, \% & 64.6 \\
\hline \multicolumn{2}{|l|}{ Marital status, $\%$ patients } \\
\hline Married & 64.6 \\
\hline Unmarried & 24.7 \\
\hline Divorced & 4.5 \\
\hline Separated & 3.4 \\
\hline Widowed & 2.8 \\
\hline \multicolumn{2}{|l|}{ Educational qualifications, $\%$ patients } \\
\hline University degree & 14.0 \\
\hline Higher certificate of secondary education & 34.3 \\
\hline 2-3 year diploma & 5.6 \\
\hline Lower certificate of secondary education & 35.4 \\
\hline Junior high-school certificate & 9.0 \\
\hline No qualifications & 1.7 \\
\hline \multicolumn{2}{|l|}{ Occupational situation, $\%$ patients } \\
\hline Employed & 56.2 \\
\hline First-time job seeker & 1.1 \\
\hline Unemployed & 7.9 \\
\hline Stopped working & 12.4 \\
\hline Student & 5.1 \\
\hline Housewife & 12.4 \\
\hline Declared unfit for work & 0.0 \\
\hline In the military or equivalent service & 0.0 \\
\hline Other & 5.1 \\
\hline Treatment cost exemption, $\%$ patients & 56.7 \\
\hline
\end{tabular}

Table 2 Clinical and health-related quality of life benefits

\begin{tabular}{|c|c|c|c|c|c|c|}
\hline & \multicolumn{3}{|c|}{ QALY } & \multicolumn{3}{|c|}{ PASI } \\
\hline & Enrolment & $\begin{array}{l}\text { Follow- } \\
\text { up }\end{array}$ & $P$-value* & Enrolment & $\Delta$ & $P$-value* \\
\hline \multirow{3}{*}{$\begin{array}{l}\text { Total } \\
\text { score }\end{array}$} & 0.6 & 0.9 & 0.000 & 21.6 & 9.0 & 0.000 \\
\hline & \multicolumn{3}{|c|}{ Pain VAS } & \multicolumn{3}{|c|}{ Itching VAS } \\
\hline & Enrolment & $\Delta$ & $P$-value* & Enrolment & $\Delta$ & $P$-value* \\
\hline $\begin{array}{l}\text { Total } \\
\text { score }\end{array}$ & 28.5 & 8.8 & 0.000 & 31.7 & 7.7 & 0.000 \\
\hline
\end{tabular}

*Paired T test.

PASI, Psoriasis Area and Severity Index; QALY, quality adjusted life years; VAS, visual analogue scale.

dropped to $€ 291.60$ per year with a difference of $€ 636.60$ $(P<0.0001)$.

With reference to direct non-medical costs prior to enrolment, each patient spent $62.3 \mathrm{~min}$ per visit in travel time to attend dermatology appointments and check-ups. During the observation period, this commitment dropped to $57.5 \mathrm{~min}$ (Table 3). Using the $€ 0.30$ unit cost per Km, a mean annual cost 
Table 3 Mean time per visit and mean travel costs per patient for appointments on an annual basis

\begin{tabular}{lll} 
& Time $(\min )$ & Cost $(€)$ \\
\hline Prior to enrolment & 56.00 & 142.71 \\
Follow-up & 54.56 & 113.28 \\
$P$-value* & & 0.573 \\
\hline
\end{tabular}

${ }^{*}$ Paired $T$ test.

of $€ 142.70$ and $€ 113.30$ per patient has been estimated prior to enrolment and subsequent to the switch to biologic therapy respectively, representing a $20.6 \%$ reduction.

Due to their illness, patients required an average of 2.3 days' assistance on a monthly basis for performing their household chores before enrolment, and 0.8 days after the switch to biologic therapy.

By attributing a cost of $€ 57.80$ per 8-h day (i.e. applying the minimum hourly wage of $€ 7.22$ ) for assistance in performing household chores, the mean annual cost amounted to €1609.49 prior to enrolment and $€ 587.98$ while on biologic therapy, with a $63.5 \%$ decrease (Table 4 ).

On average, total direct non-medical costs amounted to $€ 1752.20$ per year, of which $8.1 \%$ in travel costs and the remaining $91.9 \%$ for assistance in performing household chores. Typically, following a 1-year period of biologic treatment, direct non-medical costs decreased to $€ 701.30$ (of which $16.2 \%$ in travel costs and the remaining $83.8 \%$ for assistance in performing household chores) - corresponding to a $60.0 \%$ reduction.

Therefore, the annual direct (medical and non-medical) costs per patient amounted to $€ 4846.60$ prior to enrolment and $€ 16066.60$ in the observation year, with an $€ 11220.00$ increase following the introduction of biologic treatment that can essentially be attributed to the pharmaceutical cost.

Indirect costs included the value of productivity loss by the patient and family/friends due to the patient's illness or treatment. Prior to enrolment, patients claimed a mean of 12.4 days of lost work each year for psoriasis-related problems (Table 5). Besides, they reported on average a reduced working capacity of 18.0 days a year. Productivity loss by friends and relatives was relatively limited, being on average 7.9 days per year. In the observation period, following the switch to treatment with biologics, patients claimed that they lost on average 4.4 days' work per year for psoriasis-related problems. In addition to this, patients experienced a reduction in their capacity to work for 4.9 days per annum, while the productivity loss of friends and relatives dropped to 2.0 days a year (Table 5).

By following the human capital approach, the productivity loss calculation was based on the net national annual income per capita (which stood at $€ 29797$ according to Italian Institute of Statistics 2010 data) divided by the average number of working days (220) to obtain a value of $€ 135.40$. It was arbitrarily assumed that the reduced productive capacity amounted to $50 \%$ of normal capacity, which gave an annual indirect cost per patient of $€ 3960.10$ before enrolment and $€ 1133.40$ in the year of biologic therapy - producing a $71.4 \%$ reduction in indirect costs $(P=0.000)$ (Table 6).

With a view towards ensuring completeness of information, patients claimed to miss out on 2.6 days of leisure activities each month - a figure that dropped to 0.9 days in the observation period.

In short and on an annual basis, costs prior to enrolment were $€ 8806.60$ per patient, of which $35.1 \%$ for direct medical costs, $19.9 \%$ for direct non-medical costs and $45.0 \%$ for indirect costs. Specifically, the cost borne by the Italian NHS amounted to $€ 2166.20$ (24.6\% - Table 6). In the observation year, costs rose to $€ 17200.00$ per patient, of which $89.3 \%$ was for medical direct costs, $4.1 \%$ for non-medical direct costs and $6.6 \%$ for indirect costs. Specifically, the reduction in non-medical costs meant that $87.6 \%$ ( $€ 15073.70)$ of the costs relative to each patient was borne by the Italian Health Service. When assuming a societal perspective, the mean annual cost per patient was increased by $€ 8393.40$.

Applying the estimated benefits of biologic therapy and assuming a societal perspective, the ICER for biologic treatment of plaque psoriasis in actual clinical practice of the centres taking part in the project amounted to $€ 18634.40$ per QALY gained (Table 7). The ICER was $€ 333.60$ per PASI point, $€ 213.80$ per pain VAS point and $€ 174.40$ per itching VAS point (Table 7 ).

\section{Discussion}

The significant impact of psoriasis on society has been repeatedly demonstrated. In the USA, it was estimated to have an indirect cost, in terms of productivity loss, amounting to $\$ 1.2$ billion in 2004 . $^{8}$

The evidence available for European countries is characterized by significant variability, particularly with respect to indirect costs. In the Spanish system, the annual indirect cost for patients

Table 4 Days of household assistance per patient and corresponding cost (mean value for all centres)

\begin{tabular}{lll} 
& $\begin{array}{l}\text { Days of household assistance to perform } \\
\text { household chores per patient }\end{array}$ & $\begin{array}{l}\text { Mean annual cost of assistance to perform } \\
\text { household chores }(€)\end{array}$ \\
\hline Prior to enrolment & 27.87 & 1609.49 \\
Follow-up & 10.18 & 587.98 \\
P-value* & & 0.001 \\
\hline
\end{tabular}

${ }^{*}$ Paired $T$ test. 
Table 5 Mean number of days lost per year per patient for psoriasis-related reasons

\begin{tabular}{|c|c|c|}
\hline & Prior to enrolment & At follow-up \\
\hline Days of work (or study) lost for treatment & 12.38 & 4.36 \\
\hline Days of reduced capacity to work (or study) per patient (even if the subject did not take time off) & 17.96 & 4.02 \\
\hline $\begin{array}{l}\text { Days when the patient's condition prevented him/her from engaging in leisure activities (sports, shopping, } \\
\text { meeting friends, other hobbies, etc.) per patient }\end{array}$ & 31.62 & 10.99 \\
\hline Days of work (or study) lost by family/friends due to the patient's illness & 7.89 & 2.00 \\
\hline
\end{tabular}

Table 6 Mean total annual costs per patient (mean value over all centres, on an annual basis)

\begin{tabular}{|c|c|c|c|}
\hline Item & $\begin{array}{l}\text { Prior to } \\
\text { enrolment }\end{array}$ & At follow-up & Var \% \\
\hline Direct medical costs, $€$ & 3094.36 & 15365.38 & 396.56 \\
\hline $\begin{array}{l}\text { Proportion borne by } \\
\text { the Italian NHS, \% }\end{array}$ & 70.00 & 98.10 & 28.10 \\
\hline Direct non-medical costs, $€$ & 1752.19 & 701.28 & -59.98 \\
\hline Total direct costs & 4846.55 & 16066.66 & 231.51 \\
\hline Indirect costs, $€$ & 3960.07 & 1133.40 & -71.38 \\
\hline Total social costs & 8806.62 & 17200.06 & 95.31 \\
\hline
\end{tabular}

Table 7 Incremental cost-effectiveness ratio (ICER) - societal perspective

\begin{tabular}{lllll} 
& \multicolumn{4}{c}{ Cost per benefit } \\
\cline { 2 - 5 } & QALY & PASI & Pain VAS & Itching VAS \\
\hline Total, $€$ & 18634.42 & 333.59 & 213.80 & 174.41 \\
\hline
\end{tabular}

PASI, Psoriasis Area and Severity Index; QALY, quality-adjusted life year; VAS, visual analogue scale.

primarily on topical treatment is estimated at $€ 188.50$ per patient, ${ }^{12}$ whereas a German study (considering patients treated with topical and systemic therapy) estimated a mean value of $€ 1440.00 .^{14}$

In Italy, a cost-of-illness study conducted by Colombo et al. ${ }^{10}$ estimated that, in 2004, moderate-to-severe psoriasis generated indirect costs amounting to $32 \%$ of the mean total annual costs, with an annual total per patient equal to $€ 2681.51$. According to a CESAV (Centro di Economia Sanitaria Angelo e Angela Valenti) study, ${ }^{11}$ envisaging patients with moderate-to-severe psoriasis, the annual productivity loss was estimated to be 5.2 days per patient, with a mean indirect cost (in 2008) of $€ 224.10$.

In estimating the impact of biologic therapy, our study confirms that the direct medical costs of treatment borne by the Italian NHS rose substantially subsequent to the switch to biologic therapy. However, non-medical direct and indirect costs decreased significantly when patients were treated with biologics.

According to our estimate, indirect costs are greater compared to the Spanish and German papers. Also referring to Colombo's paper studying a sample of Italian patients, our estimate is higher $(+€ 1278.56)$.
Considering non-medical costs and indirect costs, by adopting a societal perspective the ICER is reduced from €28656.3 obtained in the third-party payer perspective, ${ }^{16}$ to $€ 18634.40$ : the significance of the impact of direct non-medical costs and, especially, indirect costs in the appraisal of the agents' value appears quite evident.

\section{Conclusion}

This article provides new insight into both direct non-medical costs and indirect costs borne by patients eligible for the biologic treatment of psoriasis, therefore contributing to the economic assessment of the societal impact of the drugs.

Direct non-medical costs and indirect costs account for nearly $65 \%$ of the total costs prior to the switch to biologic therapy (of which $69 \%$ represents productivity loss). The prescription of biologic drugs gives rise to a $68 \%$ drop in these costs, reducing indirect costs to one-third.

The results, obtained within the context of an actual clinical practice, confirm the significant social impact of psoriasis: i.e. the importance of direct non-medical costs and productivity loss, therefore the importance of adopting a societal perspective. At the same time, it stresses the effects of biologic therapy on eligible patients.

It should be noted that this was an observational study without a control group. It is therefore important to emphasize, in particular regarding ICER calculation, that a primarily conservative hypothesis was adopted by assuming that the patients' health condition and the costs incurred would remain constant without switching to biologic treatment. ${ }^{16}$

The main limitation of this research is its brief observation period, which did not allow for a comprehensive analysis of the persistent nature of benefits.

Finally, due caution should be used for the transferability of the results. As the centres taking part in the research are specialized facilities, monitored within the context of the Psocare project, they are likely to pay more attention to the appropriateness of treatment choices compared to non-specialized centres.

\section{Acknowledgements}

We wish to express our gratitude to Ray Hill, an independent medical writer, who provided medical writing support funded by Pfizer, Italy. The study was funded by Pfizer, Italy. 


\section{Author contributions}

$\mathrm{BP}$ and FS have contributed on an equal footing to the concept and design of the study, data collection, interpretation of results and drafting the article itself. BP have planned and reviewed the statistical analysis. PCP and FA have been involved in editing and critically reviewing the intellectual contents of the manuscript. All authors have read and approved the final manuscript.

\section{References}

1 Lebwohl M. Psoriasis. Lancet 2003; 361: 1197-1204.

2 Kimball AB, Gieler U, Linder D et al. Psoriasis: is the impairment to a patient's life cumulative? J Eur Acad Dermatol Venereol 2010; 24: 989-1004.

3 Russo PA, Ilchef R, Cooper AJ. Psychiatric morbidity in psoriasis: a review. Australas J Dermatol 2004; 45: 155-159.

4 Kimball AB, Jacobson C, Weiss $\mathrm{S}$ et al. The psychosocial burden of psoriasis. Am J Clin Dermatol 2005; 6: 383-392.

5 Raho G, Koleva DM, Garattini L, Naldi L. The burden of moderate to severe psoriasis: an overview. Pharmacoeconomics 2012; 30: 1005-1013.

6 Koo J. Population-based epidemiologic study of psoriasis with emphasis on quality of life assessment. Dermatol Clin 1996; 14: 485-496.

7 Meyer N, Paul C, Feneron D et al. Psoriasis: an epidemiological evaluation of disease burden in 590 patients. J Eur Acad Dermatol Venereol 2010; 24: 1075-1082.
8 The Lewin Group Inc. The Burden of Skin Diseases 2005. Prepared for the Society for Investigative Dermatology and the American Academy of Dermatology Association. The Lewin Group, Falls Church, VA, 2005.

9 Fowler JF, Duh MS, Rovba L et al. The impact of psoriasis on health care costs and patient work loss. J Am Acad Dermatol 2008; 59: 772-780.

10 Colombo G, Altomare G, Peris K et al. Moderate and severe plaque psoriasis: cost-of-illness study in Italy. Ther Clin Risk Manag 2008; 4: 559-568.

11 Valenti A, Valenti A. I costi della psoriasis vulgaris nei pazienti sottoposti a terapia sistemica: una rassegna della letteratura e una stima preliminare di costo in Italia [Italian]. Quaderni di Farmaco Economia 2008; 6: 7-15.

12 Carrascosa JM, Pujol R, Dauden E et al. A prospective evaluation of the cost of psoriasis in Spain (EPIDERMA project: phase II). J Eur Acad Dermatol Venereol 2006; 20: 840-845.

13 Griffiths CE, Clark CM, Chalmers RJ et al. A systematic review of treatments for severe psoriasis. Health Technol Assess 2000; 4: 1-125.

14 Berger K, Ehlken B, Kugland B, Augustin M. Cost-of-illness in patients with moderate and severe chronic psoriasis vulgaris in Germany. J Dtsch Dermatol Ges 2005; 3: 511-518.

15 Girolomoni G, Peserico A, Altomare A et al. Quality of life in patients with plaque psoriasis treated with biologic therapies. Clin Dermatol 2013; 1: 175-182.

16 Spandonaro F, Ayala F, Berardesca E et al. The cost effectiveness of biologic therapy for the treatment of chronic plaque psoriasis in real practice settings in Italy. BioDrugs 2014; 28: 285-295. 